Submitted:

12.06.2019

Accepted: 19.08.2019

Published: 30.09.2019

Keywords

echocardiography, musculoskeletal pain, technological innovations

\section{The use of a probe stabilizer to reduce musculoskeletal overload of ultrasound operators in routine diagnostic echocardiographic imaging}

\author{
Sjoerd Bouwmeester ${ }^{1}$, Marloes de Kleijn², Jan van Wijngaarden³, \\ Patrick Houthuizen ${ }^{1}$ \\ ${ }^{1}$ Department of Cardiology, Catharina Hospital, Eindhoven, the Netherlands \\ ${ }^{2}$ Department of Cardiology, Nij Smellinghe Hospital, Drachten, the Netherlands \\ ${ }^{3}$ Department of Cardiology, Deventer Hospital, Deventer, the Netherlands \\ Correspondence: Sjoerd Bouwmeester, Catharina Ziekenhuis Eindhoven, Michelangelolaan 2, \\ 5623 EJ Eindhoven, the Netherlands; tel.: 0031402399111, \\ e-mail: sjoerd.bouwmeester@catharinaziekenhuis.nl
}

DOI: $10.15557 / \mathrm{JoU} .2019 .0029$

\begin{abstract}
Introduction: Echocardiography is essential in the evaluation of patients with cardiovascular disease. Repetitive movements, however, expose ultrasound operators to a high risk of musculoskeletal strain injuries. Aim: We investigated to what extent a probe stabilizer could reduce repetitive movements. Materials and methods: The study population consisted of 31 male patients referred for routine transthoracic echocardiography. A good apical acoustic window was prerequisite for inclusion. Standard apical views and measurements were first recorded without using the probe stabilizer. Afterwards, the same apical views and measurements were acquired with utilization of the probe stabilizer. During the entire procedure, shoulder abduction and muscle activity of right forearm flexor and extensor muscles were recorded. To this purpose, an EMG-sensor was attached to the right lower arm and a gyroscope to the right shoulder blade. Results: Extreme right arm abduction $\left(>30^{\circ}\right)$ occurred in $58 \%$ of the time with use of the stabilizer and in $98 \%$ of the time without $(p<0.01)$. Activity of right forearm extensor muscles was $42 \%$ with and $60 \%$ without stabilizer $(p=0.04)$. For the flexor muscles these percentages were $47 \%$ and $87 \%$, respectively $(p<0.01)$. Use of the stabilizer did not affect the time needed for image acquisition $(308 \mathrm{~s}$ versus $309 \mathrm{~s}$, respectively, $p=0.46$ ). Conclusions: This study demonstrated that the use of a stabilizer during acquisition of apical views in routine transthoracic echocardiography reduces the total time of shoulder abduction and the use of the right forearm muscles, while acquisition time was
\end{abstract} not affected. 


\section{Introduction}

Echocardiography is the cornerstone of diagnostic imaging in cardiac patients due to its ease of use, cost-effectiveness, and safety. However, the disadvantage of this technique is the musculoskeletal overload of the cardiac sonographer, which results in a high incidence of workrelated injuries of up to $90 \%{ }^{(1)}$. Work-related injuries can affect the ability of sonographers to perform required occupational activities, possibly jeopardizing the quality of patient care and productivity of the ultrasound department ${ }^{(2)}$. Important causes of work-related injuries are excessive strain, repetitive motions, and duration of pressure $^{(3,4)}$. The wrist and shoulder are the most common body parts injured in sonographers ${ }^{(5,6)}$. A common static work posture during scanning contains wrist flexion and extreme shoulder abduction for a long period of time. An angle of abduction greater than $30^{\circ}$ puts the shoulder at greater risk for injury ${ }^{(7)}$. Modified scanning techniques and ergonomically designed workstations can significantly reduce the muscle activity. The objective of the current study was to investigate to what extent a probe stabilizer could reduce shoulder abduction and the muscle activity of the forearm.

\section{Methods}

\section{Study subjects}

Two Dutch cardiac ultrasound departments participated in the study, respectively the Catharina Hospital (Eindhoven, the Netherlands) and the Deventer Hospital (Deventer, the Netherlands). The study population consisted of 31 male patients referred for routine transthoracic echocardiography. A good apical acoustic window with clear endocardial border definition was a prerequisite for inclusion.

\section{Transthoracic echocardiography}

In the Catharina Hospital a Philips EPIQ 7 ultrasound system was used with a X5-1 transducer. The iRotate function was used, which made it possible to electronically rotate to the standard apical four, two, and three chamber views. In the Deventer Hospital a GE Vivid E9 ultrasound system was used with a M5S transducer. Standard apical views were recorded by manually rotating the transducer. During echocardiography patients were placed in left lateral position with the sonographer sitting at the right side of the patient. Standard apical views and measurements were first recorded without using the probe stabilizer. Afterwards, the same apical views and measurements were acquired with utilization of the probe stabilizer. During acquisition the sonographer was instructed to position both hands at the ultrasound machine, unless repositioning of the probe was necessary. During both techniques, shoulder abduction and the activity of the right forearm flexor and extensor muscles were recorded. Both the time needed to fixate the probe stabilizer, and to record apical views and measurements were registered.

\section{Probe stabilizer}

The probe stabilizer (ProbeFix ${ }^{\circledR}$, Usono, Eindhoven, the Netherlands) enables stable fixation of an ultrasound probe to the body (Fig. 1). The stabilizer consists of several parts and the probe itself is tightened into a moulded silicone ring which is fixated in the stabilizer itself. Before positioning the ultrasound probe at the ideal acoustic window, the probe is loosely connected to the stabilizer, without fixating the device to the patient's body. When the desired acoustic window is identified, the stabilizer is fixated to the chest with two straps. The probe angle can be altered in any direction, enabling probe rotation in the standard apical views.

\section{Electromyography-sensor and gyroscope}

During acquisition, the sonographer wore a small device containing an electromyography (EMG)-sensor and gyroscope (ErgoNode ${ }^{\circledR}$, Usono, Eindhoven, the Netherlands). The EMG-sensors were positioned on the inside and the outside of the forearm. A surfaced EMG was used to evaluate the right forearm extensor and flexor muscle activity during ultrasound examination, representing the wrist joint loading. In this study we did not evaluate the absolute amount of muscle activity, but attempted to identify patterns of muscle activity over time. The gyroscope was used for recording the duration of extreme shoulder abduction, which was defined as an angle greater than $30^{\circ}$.

\section{Questionnaire}

After completion of the ultrasound investigation both sonographer and patient were asked to fill out a questionnaire regarding their experience with probe stabilizer. The image quality recorded with the probe stabilizer was judged by the sonographer after finishing the examination on a 1 to 5 scale (1; very poor - 5; very good). The amount of patient discomfort with the probe stabilizer was also

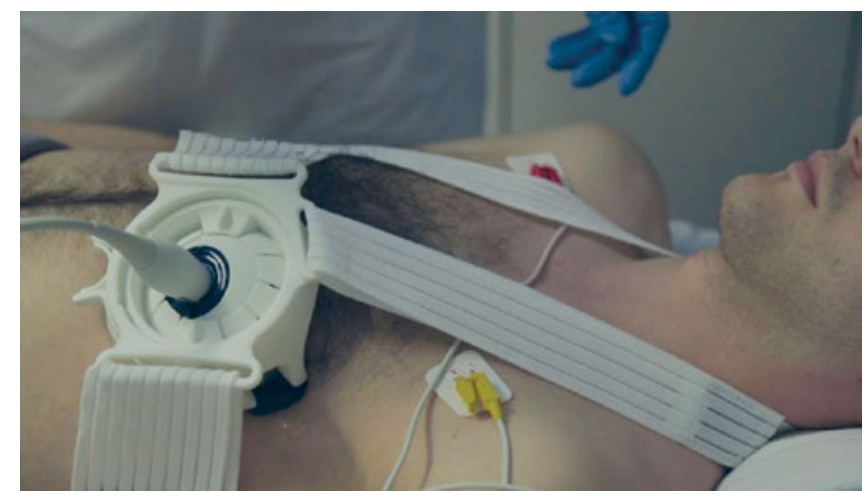

Fig. 1. Fixation of the probe stabilizer 
examined on a 1 to 5 scale (1; very uncomfortable -5 ; very comfortable).

\section{Statistical analysis}

Continuous variables are presented as mean with standard deviations. The duration of extreme right arm abduction and muscle activity are presented as percentages of the total scanning time. The nonparametric Wilcoxon signed rank test was used to compare the differences in extreme right arm abduction, muscle activity and total scanning time between recording with and without using the probe stabilizer. A $p$-value $<0.05$ was considered as statistically significant. Statistical analysis was performed using SPSS 19.0.

\section{Results}

Six cardiac sonographers participated in the study. Their professional experience ranged from 4 to 33 years. The study population consisted of 31 male patients with an average BMI of $25.4 \pm 3.1 \mathrm{~kg} / \mathrm{m}^{2}$. The average time to fixate the probe to the chest with the stabilizer took 2.7 \pm 1.3 minutes. The use of the stabilizer did not affect the time needed for image acquisition (5.1 versus 5.2 minutes, $p=0.46$ ). The average score for image quality recorded with the probe stabilizer was $3.8 \pm 1$. The patient's experience was assessed with a mean score of $4.1 \pm 1$. Extreme right arm abduction $\left(>30^{\circ}\right)$ occurred in $58 \%$ of the time with use of the stabilizer and in $98 \%$ of the time during regular scanning $(p<0.01)$. Activity of right forearm extensor muscles was $42 \%$ with and $60 \%$ without stabilizer $(p=0.04)$. For the flexor muscles these percentages were $47 \%$ and $87 \%$, respectively $(p<0.01)$ (Tab. 1 and Fig. 2).

\section{Discussion}

The present study demonstrated that the use of a probe stabilizer is a practical and easy method to relief the musculoskeletal overload in cardiac sonographers. It reduced the duration of both extreme shoulder abduction and activity of flexor muscles in the forearm with roughly $40 \%$.

The incidence of sick leave because of work-related injury is increasing in ultrasound departments ${ }^{(8,9)}$. The mechanism for injury is likely related to mechanical compression of the supraspinatus tendon with reduction of blood supply to the muscles when the arm is elevated ${ }^{(10)}$. Nowadays, departments are more motivated to minimize the risk of workrelated musculoskeletal injuries. Injuries can be reduced by optimizing work environment ergonomically (e.g. ultrasound systems, exam tables, and chairs). Moreover, work schedules could be adjusted to optimize muscle recovery time. Additionally, it is important to create awareness among sonographers about high-risk work postures such as excessive arm abduction and over-reaching ${ }^{(11-13)}$. Typically, these postures occur when sonographers do not take time to optimize their position with respect to the patient and the ultrasound system. However, the degree of overreaching and abduction are also determined by patient's body habitus. Supportive equipment can be used to contribute to minimize high-risk work postures. In the current study we investigated the advantages of using a probe stabilizer to create a hands-off workflow during transthoracic echocardiographic examination, which potentially reduces the amount of work-related injury by reducing the excessive strain, repetitive motions, and the total duration of pressure during image acquisition.

Other methods to reduce the musculoskeletal strain have been published. Firstly, left-handed scanning from the left side of the patient can be used to reduce overload ${ }^{(14)}$. Still, implementation of the left-handed scanning technique requires more time and effort to retrain, especially in aged sonographers with large experience in right-handed scanning. Furthermore, an articulating support arm system was developed ${ }^{(15)}$. It is an articulating arm, designed to support the arm of the sonographer in a position to hold the transducer against the patient's chest. Though, the system is intended for sonographers who scan with the left hand. The authors observed a significant reduction in muscle activity and shoulder abduction. The advantage of the probe stabilizer used in the current study is that after positioning the probe, a hands-off acquisition of views and measurements can be applied. Additionally, it has shown to be useful for stress echocardiography during ergometer tests in supine and upright positions ${ }^{(16)}$.

This study was performed in two different hospitals in the Netherlands. One of the hospitals $(\mathrm{CH})$ used the iRotate method in combination with the probe stabilizer, which automatically rotates for the different apical windows, whereas the other hospital (DH) rotated the probe manually. We observed that the benefit on musculoskeletal load was higher when the probe stabilizer was combined with the iRotate method, which was used in 18 patients. With this method in combination with the stabilizer, extreme shoulder abduction occurred only in $36 \%$ of the time. With manual rotation extreme abduction occurred in $81 \%$ of the

Tab. 1. Percentage of shoulder abduction and the muscle activity of the forearm during scanning with and without the use of the probe stabilizer

\begin{tabular}{|l|c|c|c|}
\hline & $\begin{array}{c}\text { With probe } \\
\text { stabilizer } \\
\mathbf{n = 3 1}\end{array}$ & $\begin{array}{c}\text { Without } \\
\text { probe } \\
\text { stabilizer } \\
\boldsymbol{n}=\mathbf{3 1}\end{array}$ & P-value \\
\hline $\begin{array}{l}\text { Extreme shoulder } \\
\text { abduction over time (\%) }\end{array}$ & 58 & 98 & $<0.01$ \\
\hline $\begin{array}{l}\text { Activity of forearm exten- } \\
\text { sor muscles over time (\%) }\end{array}$ & 42 & 60 & 0.04 \\
\hline $\begin{array}{l}\text { Activity of forearm flexor } \\
\text { muscles over time (\%) }\end{array}$ & 47 & 87 & $<0.01$ \\
\hline $\begin{array}{l}\text { Time to fixate probe } \\
\text { stabilizer (minutes) }\end{array}$ & $2.7 \pm 1.3$ & - & 0.46 \\
\hline $\begin{array}{l}\text { Time image acquisition } \\
\text { (minutes) }\end{array}$ & 5.2 & 5.1 & \\
\hline
\end{tabular}




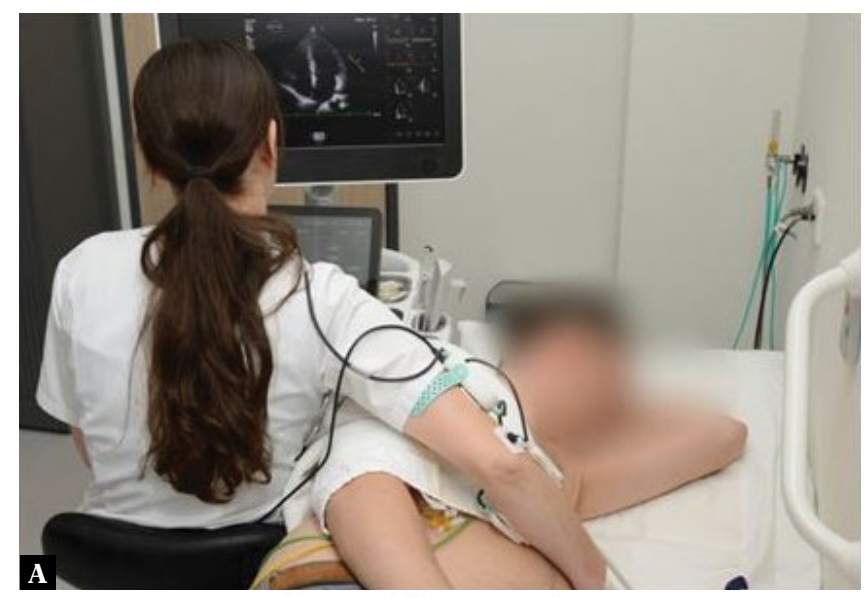

Forearm flexor muscles

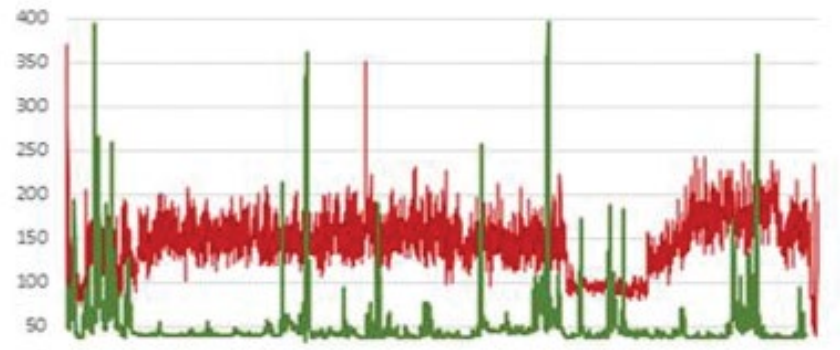

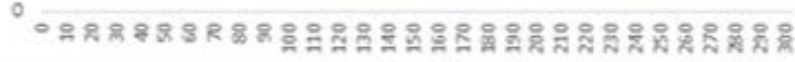

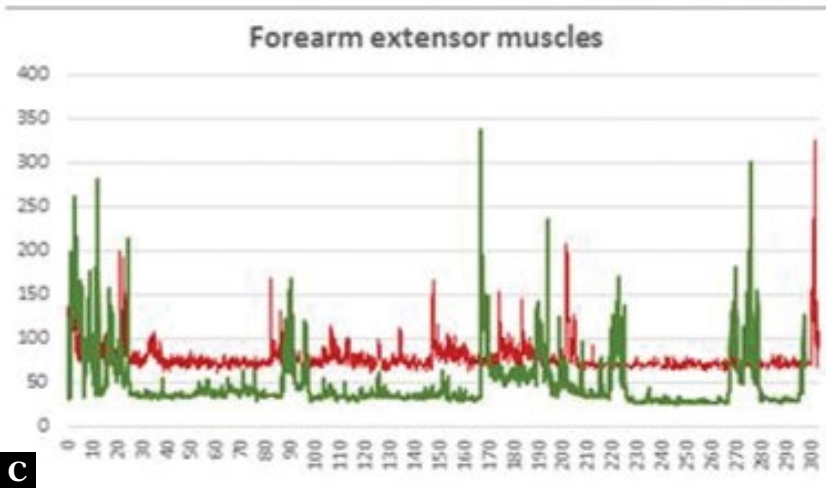

time $(p<0.01)$. The percentage of flexor muscle activity in the forearm was $32 \%$ versus $64 \%(p<0.01)$, respectively.

\section{Limitations}

There are some limitations to this study. Firstly, this was a pilot study with a short follow-up which not registered the number of actual work-related injuries. Secondly, we only collected data on movements of shoulder and wrist because these are the body parts most prone for injuries ${ }^{(5,6)}$. In future studies it should be considered to also analyse the effect on the neck, back, and hands, since these are also often mentioned locations of pain and discomfort ${ }^{(3,17)}$. Moreover, our study included only men with a good apical acoustic window. Therefore, it remains unsure if the probe stabilizer can be applied in women and in patients with other body characteristics such as obesity. Nevertheless, we

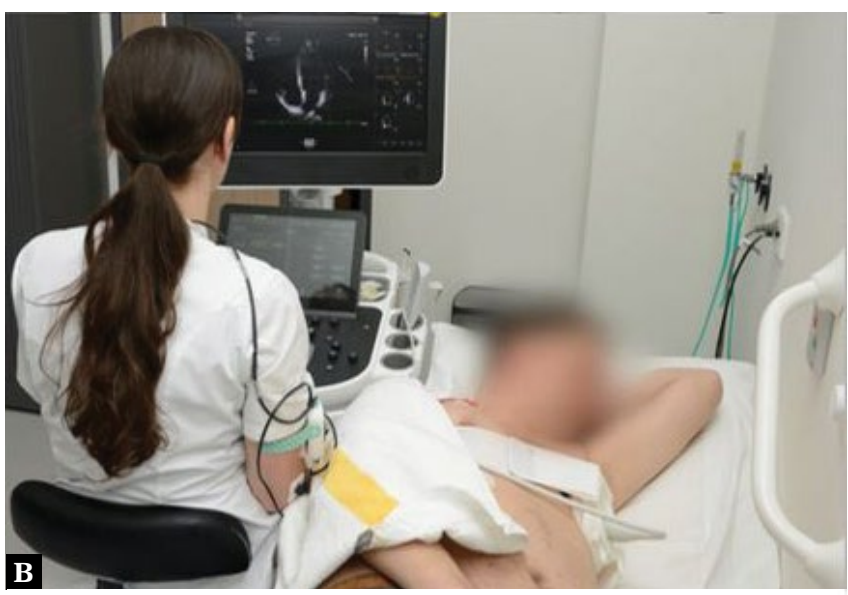

Shoulder abduction

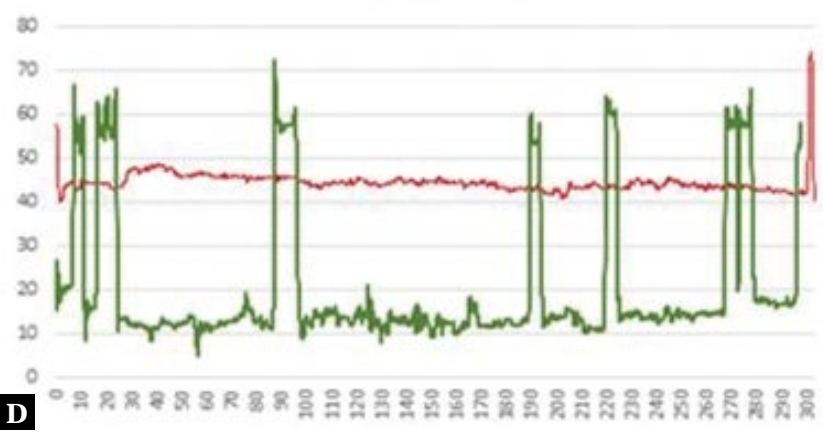

Fig. 2. A. Extreme right shoulder abduction during standard scanning. B. The position of the right arm after fixation of the probe stabilizer. C. EMG of the forearm flexor and extensor muscles. Red: conventional scanning, Green: scanning with the probe stabilizer. D. Degrees of shoulder abduction. Red: conventional scanning, Green: scanning with the probe stabilizer

expect the probe stabilizer to reduce work related injuries because we assume the stabilizer can be applied at least in $30 \%$ of patients in our echo department.

\section{Conclusion}

This study demonstrated that the use of a probe stabilizer by cardiac sonographers during routine transthoracic echocardiography reduces the total time of shoulder abduction and the use of the right forearm muscles. The probe stabilizer is therefore a promising tool in reducing work-related injury of sonographers.

\section{Conflict of interest}

The authors declare no conflicts of interest in this paper. 


\section{References}

1. Evans K, Roll S, Baker J: Work-related musculoskeletal disorders (WRMSD) among registered diagnostic medical sonographers and vascular technologists: a representative sample. J Diagn Med Sonogr 2009; 25: 287-299.

2. Escorpizo R: Understanding work productivity and its application to workrelated musculoskeletal disorders. Int J Ind Ergon 2008; 38: 291-297.

3. Necas M: Musculoskeletal symptomnatology and repetitive strain injuries in diagnostic medical sonographers. J Diagn Med Sonogr 2006; 12: 266-273.

4. Punnett L, Wegman DH: Work-related musculoskeletal disorders: the epidemiologic evidence and the debate. J Electromyogr Kinesiol 2004; 14: 13-23.

5. Pike I, Russo A, Berkowitz J, Baker JP, Lessoway VA: The prevalence of musculoskeletal disorders among diagnostic medical sonographers. J Diagn Med Sonogr 1997; 13: 219-227.

6. Evans K, Roll SC, Hutmire C, Baker JP: Factors that contribute to wristhand-finger discomfort in diagnostic medical sonographers and vascular technologists. J Diagn Med Sonogr 2010; 26: 121-129.

7. Punnett L, Fine LJ, Keyserling WM, Herrin GD, Chaffin DB: Shoulder disorders and postural stress in automobile assembly work. Scand J Work Environ Health 2000; 26: 283-291.

8. Friesen MN, Friesen R, Quanbury A, Arpin S: Musculoskeletal injuries among ultrasound sonographers in rural Manitoba: a study of workplace ergonomics. AAOHN J 2006; 54: 32-37.

9. Muir M, Hrynkow P, Chase R, Boyce D, McLean D: The nature, cause, and extent of occupational musculoskeletal injuries among sonogra- phers: recommendations for treatment and prevention. J Diagn Med Son 2004; 20: 317-325.

10. Garg A, Hegmann K, Kapellusch J: Short-cycle overhead work and shoulder girdle muscle fatigue. Int J Ind Ergon 2006; 36: 581-597.

11. Kroemer K, Grandjean E: Fitting the Task to the Human. Taylor \& Francis, London 1997.

12. Village J, Trask C: Ergonomic analysis of postural and muscular loads to diagnostic sonographers. Int J Ind Ergon 2007; 37: 781-789.

13. Jakes C: Sonographers and occupational overuse syndrome: cause, effect, and solutions. J Diagn Med Sonogr 2001; 17: 312-320.

14. Coffin CT: Work-related musculoskeletal disorders in sonographers: a review of causes and types of injury and best practices for reducing injury risk. Rep Med Im 2014; 7: 15-26.

15. Sommerich CM, Jing L, Nagavarupu S, Palmer D, Ngo S, Zaid R et al.: A pilot study of an articulating support arm system for reducing sustained posture and muscular effort while performing echocardiograms. Proceed Human Factors Ergonom Soc Ann Meet 2016; 60: 1492-1494.

16. Salden OAE, van Everdingen WM, Spee R, Doevendans PA, Cramer MJ: How I do it: Feasibility of a new ultrasound probe fixator to facilitate high quality stress echocardiography. Cardiovasc Ultrasound 2018; 16:6.

17. Burnett DR, Campbell-Kyureghyan NH: Quantification of scan-specific ergonomic risk-factors in medical sonography. Int J Ind Ergon 2010; 40: 306-314. 